\title{
Clinical experience of conversion from cyclosporine to tacrolimus prolonged-release in stabilized kidney transplant patients
}

\author{
Karel Krejcia, Josef Zadrazila, Eva Lackova ${ }^{b}$, Zuzana Zilinskac, Robert Roland ${ }^{d}$, Ivana Dedinska ${ }^{e}$
}

\begin{abstract}
Background and Aims. The CONCERTO study results showing the beneficial effects of conversion from cyclosporine to tacrolimus prolonged-release (tacrolimus PR) in stabilised patients after kidney transplantation, were first published in 2011. This communication describes our first experience of conversion from cyclosporine to tacrolimus PR in stabilised kidney transplant patients. The aim was to determine whether it could be used in routine clinical practice in the Czech and Slovak Republics.
\end{abstract}

Methods. Evaluation was carried out at five transplantation centres in the Czech Republic and Slovakia. In all participating Centres, the drug conversion was conducted according to the ICH/GCP guidelines. A total of 104 patients stabilised after kidney transplantation were converted from maintenance therapy with cyclosporine to treatment with tacrolimus PR. The data were collected 26 weeks after the switch. The primary endpoint was change in kidney graft function measured from the estimated glomerular filtration rate (GFR). The effect of conversion on blood pressure, metabolic parameters and cosmetic changes was also recorded. Special attention was paid to the safety and tolerability of treatment with tacrolimus PR.

Results. GFR increased after six months by $10 \%(P=0.040)$. In addition a significant decrease in serum creatinine and triglycerides level was found together with major reduction in the incidence and severity of gingival hyperplasia and hirsutism. $3 \%$ of patients developed new onset of diabetes mellitus. Otherwise, the switch was very well-tolerated, without serious adverse events or acute rejections.

Conclusion. Conversion from cyclosporine to tacrolimus PR was shown to be a safe therapeutic alternative with patient benefits.

Key words: tacrolimus prolonged-release, cyclosporine, immunosuppressive conversion, kidney transplantation

Received: December 14, 2015; Accepted with revision: April 26, 2016; Available online: May 12, 2016

http://dx.doi.org/10.5507/bp.2016.026

${ }^{a}$ Department of Internal Medicine III - Nephrology, Rheumatology and Endocrinology, University Hospital Olomouc, Czech Republic

${ }^{\circ}$ Transplant Centre, University Hospital F.D. Roosevelta, Banska Bystrica, Slovak Republic

'Department of Urology with the Centre for kidney transplant, Academician Derer University Hospital, Bratislava, Slovak Republic

${ }^{d}$ Fresenius Medical Care Dialysis, Kosice, Slovak Republic

${ }^{e}$ Department of Surgery and Transplant Centre, University Hospital Martin, Slovak Republic

Corresponding author: Karel Krejci, e-mail: karel.krejci@fnol.cz

\section{INTRODUCTION}

In 2011, the results of the CONCERTO study ${ }^{1}$ were the first published, showing the beneficial effects of conversion from cyclosporine to tacrolimus PR (brand name ADVAGRAF $^{\circledR}$ ) in stabilised patients after kidney transplantation. In six months of follow-up, the positive effects of conversion on kidney function and the undesirable side-events of cyclosporine treatment were observed. The study showed that kidney functions remained unchanged and the conversion to the new immunosuppressive drug was very well tolerated by patients with decrease in existing hirsutism and gingival hyperplasia. There were only minor corrections in elevated blood pressure and dyslipidaemia.

These positive results raised the interest of transplantation specialists in the Czech Republic and Slovakia and to determine whether these results could be achieved in routine clinical practice, they decided to convert their stabilised patients from immunosuppressive treatment with cyclosporine to treatment with tacrolimus PR and evaluate results of this conversion after 26 weeks. Described below are the results of this follow-up period.

\section{MATERIALS AND METHODS}

This type of research does not require approval of Institutional Review Boards or registration as a clinical trial. Evaluation was carried out at five transplantation centres in Czech Republic and Slovakia, on all participating sites the drug conversion was conducted according to the ICH/GCP guidelines. A total of 104 patients were enrolled in the project, 65 men and 39 women. The mean age was 55.0 years and mean time since the kidney transplantation was 8.3 years for the whole cohort (but for $37 \%$ of patients this period was longer than 10 years). For $92 \%$ of patients this was their first transplantation and the remaining $8 \%$ their second transplantation. No patient had more than two transplantations. Principal 
Table 1. Principal demographic data.

\begin{tabular}{|c|c|c|c|c|c|}
\hline Parameter & Number & $\begin{array}{c}\text { Age } \\
\text { (years) }\end{array}$ & $\begin{array}{l}\text { Height } \\
(\mathrm{cm})\end{array}$ & $\begin{array}{l}\text { Weight } \\
(\mathrm{kg})\end{array}$ & $\begin{array}{c}\text { Time since transplantation } \\
\text { (months) }\end{array}$ \\
\hline Overall & 104 & & & & \\
\hline $\begin{array}{l}\text { Mean } \\
(\min ; \max )\end{array}$ & & $\begin{array}{c}55 \\
(20 ; 77)\end{array}$ & $\begin{array}{c}172 \\
(151 ; 191)\end{array}$ & $\begin{array}{c}79 \\
(44 ; 112)\end{array}$ & $\begin{array}{c}100 \\
(1 ; 304)\end{array}$ \\
\hline Men & 65 & & & & \\
\hline $\begin{array}{l}\text { Mean } \\
(\min ; \max )\end{array}$ & & $\begin{array}{c}54.8 \\
(20 ; 77) \\
\end{array}$ & $\begin{array}{c}177 \\
(162 ; 191)\end{array}$ & $\begin{array}{c}83 \\
(64 ; 103) \\
\end{array}$ & $\begin{array}{c}104 \\
(1 ; 304) \\
\end{array}$ \\
\hline Women & 39 & & & & \\
\hline $\begin{array}{l}\text { Mean } \\
\text { (min; max) }\end{array}$ & & $\begin{array}{c}55.3 \\
(24 ; 72)\end{array}$ & $\begin{array}{c}164 \\
(151 ; 178)\end{array}$ & $\begin{array}{c}73 \\
(44 ; 112)\end{array}$ & $\begin{array}{c}93 \\
(1 ; 206)\end{array}$ \\
\hline
\end{tabular}

Min, minimum; Max, maximum.

demographic data are shown in Table 1. No patient interrupted treatment and hence data from all 104 patients were available.

The most common diagnoses which led to kidney failure and subsequent transplantation were chronic glomerulopathy (29\%), followed by chronic interstitial nephritis or hypertensive nephrosclerosis (20\%), autosomal dominant polycystic kidney disease (13\%) and diabetic nephropathy (11\%). The rest had kidney failure because of other causes or of unknown origin.

Prior to conversion, all 104 patients were treated with cyclosporine, in most cases in triple combination with mycophenolate and corticosteroids (67\% of patients), or only in combination with mycophenolate $(29 \%)$. Only one patient was treated by cyclosporine alone. $3 \%$ of patients used less the common combination of cyclosporine and azathioprin. Patients were converted from cyclosporine to tacrolimus PR, indication for conversion, conversion rate and subsequent dose of tacrolimus PR as well as frequency of outpatient controls were on physician discretion only. The mean dose of cyclosporine before conversion was $170.2 \mathrm{mg}$ and mean trough blood level was $121.4 \mathrm{\eta g} /$ $\mathrm{mL}$. The mean conversion ratio from cyclosporine to tacrolimus PR was 31:1 (mg:mg), however with marked differences between Centres, from the lowest ratio of 22:1 up to the highest ratio of 42:1. Mean cyclosporine dose calculated by $\mathrm{kg}$ of body weight was $2.21 \mathrm{mg} / \mathrm{kg}$, mean tacrolimus PR dose calculated by $\mathrm{kg}$ of body weight was $0.07 \mathrm{mg} / \mathrm{kg}$, in this case the conversion ratio was $31.6: 1$, i.e. about 32:1. The dosage of mycophenolate was not routinely changed after conversion. For each patient the dose depended on the clinical status and laboratory findings or on blood levels of mycophenolate and was left completely to the discretion of respective sites. Information on possible change of the dose of mycophenolate was not recorded in the follow-up file.

After the conversion to tacrolimus PR, a number of selected parameters were collected in accordance with the usual local post conversion protocol. In particular, changes in renal graft function by assessment of GFR estimated by MDRD4 equation and serum creatinine level. In addition, the effect of conversion on blood pressure, development of diabetes mellitus, parameters of lipid spectrum, changes in liver function tests and cosmetic effects were recorded. Special attention was given to the safety and tolerability of the conversion during the first six months of the follow-up.

\section{Statistical analysis}

The data were analysed using IBM SPSS Statistics for Windows, Version 20.0 (Armonk, NY: IBM Corp., USA). The data were described as means $\pm \mathrm{SD}$, and frequency distributions. The Chi-square test was used for categorical variables. For normative data the two-tailed t-test was used and significance level was $P<0.05$.

\section{RESULTS}

\section{Dose of tacrolimus PR}

The mean dose of tacrolimus PR administered immediately after conversion was $5.49 \pm 2.06 \mathrm{mg}$, i.e. $0.069 \mathrm{mg} /$ $\mathrm{kg}$ body weight. As tacrolimus belongs to drugs with narrow therapeutic index, the subsequent doses were titrated in accordance with tacrolimus levels in blood at the end of 24-hour dosing interval (trough levels). At the end of the six months follow-up period, the mean dose of tacrolimus PR was $3.66 \pm 1.96 \mathrm{mg}$, i.e. $0.046 \mathrm{mg} / \mathrm{kg}$ body weight. This decrease in dose was accompanied by similar decrease in tacrolimus blood levels, from $8.86 \pm 4.50 \eta \mathrm{g} / \mathrm{mL}$ at week 4 to $6.47 \pm 2.49 \eta \mathrm{g} / \mathrm{mL}$ at the end of the six-months period. The dynamics of decrease of both tacrolimus PR mean doses and mean tacrolimus blood levels is shown in Fig. 1. Changes in both parameters between conversion and week 26 were statistically significant.

\section{Kidney function}

The primary endpoint was the change in GFR at 26 weeks after the conversion compared to baseline. Renal function was also expressed by serum creatinine concentration. The mean level of GFR prior to conversion to tacrolimus PR was $0.791 \pm 0.39 \mathrm{~mL} / \mathrm{s}, 26$ weeks after the conversion the filtration rate increased by $0.083 \mathrm{~mL} / \mathrm{s}$ to the final $0.874 \pm 0.39 \mathrm{~mL} / \mathrm{s}, P=0.040,95 \%$ CI between -0.153 and -0.013 . Mean creatinine level decreased by 17.1 $\mu \mathrm{mol} / \mathrm{L}$ from the original value of $155.5 \pm 53.2 \mu \mathrm{mol} / \mathrm{L}$ at conversion to a final $138.4 \pm 50.4 \mu \mathrm{mol} / \mathrm{L}$ at the end of follow-up; $P=0.0001,95 \%$ CI between 8.865 and 25.359 . 


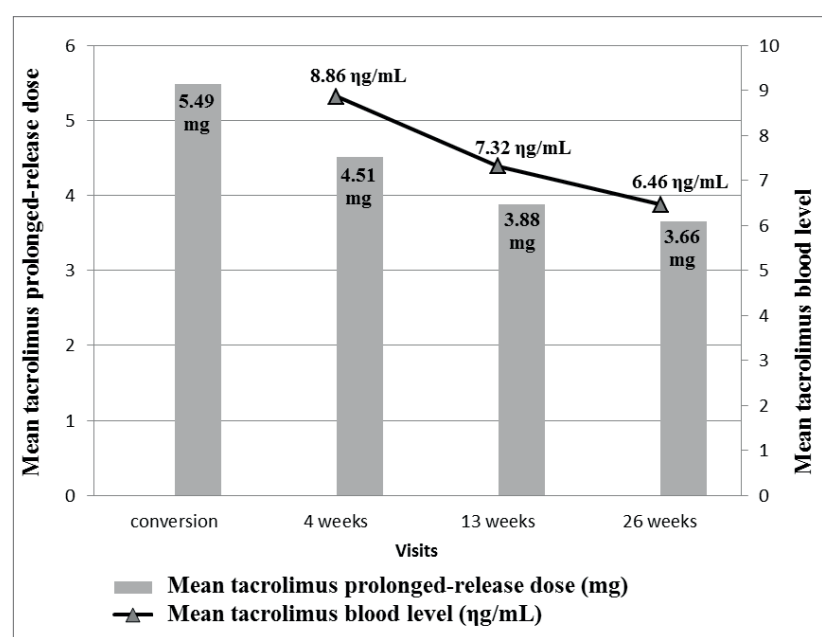

Fig. 1. Changes in mean tacrolimus prolonged-release dose and mean tacrolimus blood levels during the 26-weeks follow-up.

The changes in parameters corresponding to kidney function were statistically significant.

\section{The effect on hypertension}

At the start of follow-up, $93 \%$ of the subjects $(n=$ 97) were assessed as being hypertensive. This remained unchanged during the whole period. However after the 26 weeks of observation, a non-significant decrease in systolic blood pressure from 134 points of mercury to 132 points was recorded as well as a decrease in diastolic blood pressure from 81 to 77 points of mercury. There was also an important reduction of antihypertensive medication. At the beginning of the monitoring, 48\% (n $=50$ ) of patients were treated by a combination of three antihypertensives, after 26 weeks this figure dropped to $34 \%(n=35)$. The difference was consistent with $14 \%$ of patients who were successfully switched to combination treatment with two antihypertensives only.

\section{Dyslipidaemia}

At the beginning of the follow-up, a clinically significant dyslipidaemia was diagnosed in $69 \%(n=72)$ of patients. At the end of observation period the corresponding figure was $70 \%(n=73) .80 \%$ of patients $(n=58)$ diagnosed with dyslipidaemia at any time during observation period were treated by hypolipidemic drugs. In comparison with baseline, a statistically significant decrease in mean triglyceride level was observed $(P=0.044,95 \% \mathrm{CI}$ between 0.007 and 0.433 ). Mean LDL-cholesterol level decreased insignificantly ( $P=0.68 ; 95 \%$ CI between -0.120 and 0.182 ). Changes in the parameters of lipid metabolism are shown in Table 2.

\section{Diabetes mellitus}

Mean level of glycaemia at the start of the observation period was $5.65 \mathrm{mmol} / \mathrm{L}$, and increased insignificantly at the end of the follow-up to $5.74 \mathrm{mmol} / \mathrm{L}$. At the start of the monitoring, $21 \%$ of all patients $(n=22)$ had an already diagnosed diabetes mellitus. This subgroup was treated either by dietary regimen (in $2 \%$ of patients), by oral antidiabetics (in $5 \%$ of patients), or insulin (14\% of patients). Mean daily dose of insulin did not substantially change during the 26 weeks of follow-up period, as it was 41.3 I.U. at the beginning and 41.1 I.U. at the end. After 26 weeks, the number of patients with diagnosed diabetes increased by $7 \%$ to $28 \%(n=29)$ of the whole cohort. Some of these new patients were compensated by dietary regimen only. The number increased from $2 \%$ to a final of $8 \%(n=8)$. The number of patients requiring insulin therapy increased as well (by 3\%) and by the end of the observation represented $17 \%(n=18)$. The level of $\mathrm{HbA1c}$ decreased from the original value of $5.38 \%$ to a final of $5.15 \%$. Mean changes in the parameters of glycide metabolism are shown in Table 2.

\section{Hirsutism and gingival hyperplasia}

Hirsutism was recorded in $13 \%$ of patients $(n=14)$ treated with cyclosporine, two ( $2 \%$ of the total cohort) were assessed as severe. After conversion to tacrolimus PR and six months of therapy, the excessive body hair disappeared in most patients, with hirsutism seen in only $4 \%$ of patients $(n=4)$; all cases were mild forms. Similar development was observed with gingival hyperplasia, which before conversion afflicted $30 \%$ of all patients ( $n$ $=31), 12 \%$ of cases $(n=12)$ were diagnosed as severe. After six months of treatment gingival hyperplasia generally retreated and persisted in only $2 \%(n=2)$ of patients in a mild form.

\section{Other evaluated parameters}

At both the beginning and the end of observation, $\mathrm{BMI}$, pulse frequency and other vital functions were recorded in all patients. Blood samples were taken and levels of selected biochemical parameters - ALT, AST, bilirubin - were assessed to evaluate potential hepatotoxicity. During the follow-up period no major change in any

Table 2. Parameters of lipid and glycide metabolism before and after conversion.

\begin{tabular}{lcccc}
\hline Parameter & $\begin{array}{c}\text { LDL cholesterol } \\
(\mathrm{mmol} / \mathrm{L})\end{array}$ & $\begin{array}{c}\text { Triglycerides } \\
(\mathrm{mmol} / \mathrm{L})\end{array}$ & $\begin{array}{c}\text { Glycemia } \\
(\mathrm{mmol} / \mathrm{L})\end{array}$ & $\begin{array}{c}\text { HbA1c } \\
(\%)\end{array}$ \\
mean $\pm \mathrm{SD}$ & $2.48 \pm 0.74$ & mean \pm SD & $5.65 \pm 1.32$ & $5.38 \pm 2.43$ \\
Before conversion & $2.29 \pm 0.65$ & $1.79 \pm 1.23$ & $5.74 \pm 1.17$ & $5.15 \pm 1.55$ \\
After 26 weeks & n.s. & 0.044 & n.s. & n.s. \\
Statistical significance & & & & \\
\hline
\end{tabular}

Data are mean \pm standard deviation (SD). LDL, low density lipoprotein; HbA1c, glycated haemoglobin. 
Table 3. Selected clinical and biochemical parameters before and after conversion.

\begin{tabular}{lccccc}
\hline Parameter & BMI & $\begin{array}{c}\text { Pulse frequency } \\
(\mathrm{min}) \\
\text { mean } \pm \text { SD }\end{array}$ & $\begin{array}{c}\text { ALT } \\
(\mu \mathrm{kat} / \mathrm{L}) \\
\text { mean } \pm \text { SD }\end{array}$ & $\begin{array}{c}\text { AST } \\
(\mu \mathrm{kat} / \mathrm{L}) \\
\text { mean } \pm \text { SD }\end{array}$ & $\begin{array}{c}\text { Bilirubin } \\
(\mathrm{mmol} / \mathrm{L}) \\
\text { mean } \pm \text { SD }\end{array}$ \\
\hline Before conversion & $26.8 \pm 4.5$ & $74 \pm 7.0$ & $0.42 \pm 0.19$ & $0.42 \pm 0.18$ & $10.82 \pm 5.50$ \\
After 26 weeks & $26.9 \pm 4.6$ & $73 \pm 7.0$ & $0.40 \pm 0.19$ & $0.39 \pm 0.14$ & $11.11 \pm 8.29$ \\
Statistical significance & n.s. & n.s. & n.s. & n.s. & n.s. \\
\hline
\end{tabular}

Data are means \pm standard deviation (SD). BMI, Body Mass Index; ALT, alanine aminotransferase; AST, aspartate aminotransferase.

of these parameters was recorded. The mean results of clinical and biochemical records are shown in Table 3.

\section{Safety and tolerance}

During the 26 weeks of follow-up, no case of subject discontinuation for lack of efficacy or adverse event was recorded. All patients finished six months follow-up on tacrolimus PR and continued to be treated further. No investigator reported any unexpected drug reaction or drug related adverse event. Patients tolerated the treatment with tacrolimus PR well. In 7\% of patients, new onset of diabetes was recorded but $5 \%$ of these were compensated by diet regimen only.

\section{Assessment of the treatment by physicians}

At the end of 26-week follow-up, the physicians were asked to categorise individual patients as improved, unchanged or worse. If the patient was worse, the reason was investigated. This assessment was done twice, from the efficacy as well as from the safety and tolerance point of view. In the efficacy assessment, $56 \%$ of patients $(n=$ $58)$ were evaluated as improved, $38 \%$ patients $(n=40)$ showed no substantial change and in $6 \%$ of cases $(n=$ 6 ) the status was described as worse. In all six cases, the cause was the slow decline in renal function and gradual deterioration of the graft. These cases of deterioration were perceived by investigators to be caused by the age of the graft (i.e. long time after transplantation) and/or patient comorbidities. In no case was the reason for worse efficacy outcome linked to the conversion to tacrolimus PR. No sudden impairment in kidney graft function which could be linked to the acute rejection was recorded during the 26-weeks follow-up. There was also no case of graft loss during this period. Improved tolerance of the treatment after conversion was recorded in $48 \%$ of patients, $46 \%$ of patients remained without substantial change and in $6 \%$ of cases a worse tolerance to treatment mainly due to mild form of tremor and diarrhoea was recorded.

\section{DISCUSSION}

The results of this follow-up in routine clinical conditions confirmed the results of the clinical study CONCERTO, namely efficacy and tolerance of conversion from cyclosporine to tacrolimus PR. The switch to a new immunosuppressive therapy went smoothly, without any acute rejection or any significant adverse event linked to the treatment.
The primary evaluated parameter was kidney function assessed as the change in GFR and changes in serum creatinine concentration. In the CONCERTO study, GFR remained stable after conversion (which was assessed as a success of conversion), whereas in this noninterventional monitoring the GFR increased by more than $10 \%$. Supporting this result the serum creatinine concentration in this follow-up decreased by $11 \%$, whereas in CONCERTO study it remained unchanged after conversion. Our observation thus suggested a possible protective effect on renal function after conversion from cyclosporine to tacrolimus PR not shown in CONCERTO study. One explanations is the smaller number of patients enrolled in this local follow-up (about one third of the number enrolled in the clinical study CONCERTO). The result of this single monitoring on GFR should therefore be considered in conjunction with CONCERTO study results.

The other parameters were very similar to the results of the clinical study CONCERTO. The non-interventional follow-up also showed gradual decrease in tacrolimus PR dose as well as blood levels. It is of interest that although in this project no fixed border lines for tacrolimus blood levels were specified, mean levels in this observation were similar at week $26(6.47 \mathrm{ng} / \mathrm{mL})$ compared to mean levels in the CONCERTO study $(7.20 \mathrm{\eta g} / \mathrm{mL})$. Mean triglycerides levels decreased significantly (by $14 \%$ ) and mean LDL-cholesterol levels also dropped (by 8\%), although not significantly. This correlates with the positive effect on dyslipidaemia in the CONCERTO study. After conversion, a non-significant decrease in systolic blood pressure was recorded (by 1\%) together with statistically significant drop in diastolic blood pressure (by 5\%). This reflects the observed decrease of antihypertensive medication. In patients with resistant arterial hypertension, the administration of tacrolimus prolonged-release could have a positive clinical effect and in this situation may be preferred over the administration of cyclosporine. In a small number of patients, new onset of diabetes mellitus was also seen.

Both in CONCERTO study as well as in the local follow-up, a substantial improvement in hirsutism and gingival hyperplasia was documented after the interruption of treatment with cyclosporine. In most cases, this cosmetic complication disappeared or improved significantly. 


\section{CONCLUSION}

Conversion from cyclosporine to tacrolimus PR went smoothly in stabilised patients who were treated by cyclosporine for many years following kidney transplantation. The mean kidney functions improved as well as other clinical and laboratory parameters such as decrease in the use of antihypertensive medication, diastolic blood pressure and triglyceride blood levels. The so-called cosmetic side effects of cyclosporine (hirsutism, gingival hyperplasia) nearly disappeared. Most of the results of our observational project performed in routine clinical conditions confirmed the results of the CONCERTO study. Surprising was the observed improvement in GFR and decrease in serum creatinine levels.

\section{ABBREVIATIONS}

Tacrolimus PR, tacrolimus prolonged-release; ICH/ GCP, International Council for Harmonisation/Good Clinical Practice; GFR, glomerular filtration rate; MDRD4, 4-variable Modification of Diet in Renal Disease; SD, standard deviation; CI, confidence interval; LDL, low density lipoprotein; I.U., International Unit; HbA1c, glycated haemoglobin; BMI, Body Mass Index; ALT, alanine aminotransferase; AST, aspartate aminotransferase.

Author contributions: All authors contributed equally to preparing the manuscript.

Conflict of interest statement: None declared.

\section{REFERENCE}

1. Rostaing L, Sánchez-Fructuoso A, Franco A, Glyda M, Kuypers DR, Jaray J. Conversion to tacrolimus once-daily from ciclosporin in stable kidney transplant recipients: a multicenter study. Transpl Int 2012;25(4):391-400. 